\title{
Enhancing cooling performance via airflow temperature fluctuations
}

\author{
Žiga Lampret ${ }^{1, *}$, Gorazd Krese ${ }^{2}$, and Matjaž Prek ${ }^{l}$ \\ ${ }^{1}$ University of Ljubljana, Faculty of Mechanical Engineering, Ljubljana SI-1000, Slovenia \\ ${ }^{2}$ Korona inženiring d.d., Ljubljana SI-1000, Slovenia
}

\begin{abstract}
In ventilated and air-conditioned indoor environment, air movement substantially impacts thermal sensation and comfort of occupants from the point of view of whole body and local thermal sensation. Skin temperature and its rate of change are important factors for thermal sensation. Both are affected by the airflow velocity and temperature changes around the body which causes skin temperature fluctuations and changes in convective heat transfer. In this study the impact of temperature fluctuations in airflow on human thermal sensation was examined. For the purposes of the study, an air handling unit was designed for generating airflows with temperature fluctuations and used in a subjective experiment. The experimental study indicates that temperature fluctuations possibly influence the human perception of air movement with a distinct cooling effect.
\end{abstract}

\section{Introduction}

In developed countries people spend between $80 \%$ and $90 \%$ of their time indoors [1]. Ventilation and airconditioning systems in buildings therefore need to be able to create healthy and comfortable environment for the occupants. Inside built environment air movement can substantially impact thermal sensation and comfort of occupants from the point of view of whole body and local thermal sensation. Satisfactory understanding of the affecting factors on the perception of air movement is crucial for the enhancement of indoor thermal sensation.

\subsection{Fanger's draft model}

In the past 70-80 years numerous thermal comfort studies examined the effect of air movement on the human thermal sensation. First studies focused on the effect of the velocity and air temperature which was the basis for the development of the first draft model for assessment of the predicted percentage of dissatisfied due to draft [2-4]. Impact of the fluctuating air velocity was also noted to have an impact on the human thermal sensation. Fanger et al. expanded the draft model incorporating impact of the airflow turbulent behaviour with turbulence intensity $T u$ [5]:

$$
T u=\frac{\sqrt{\bar{v}_{a}^{\prime 2}}}{\bar{v}_{a}}
$$

where $\bar{v}_{a}^{\prime}$ is the instantaneous air velocity $(\mathrm{m} / \mathrm{s}), \overline{\mathrm{v}}_{\mathrm{a}}$ is the mean velocity and $\sqrt{\bar{v}_{a}^{2}}$ is the velocity standard deviation.
In the draft model Fanger adopted Hensel's conclusion about thermoreceptor responses. According to Hensel [6] two different thermoreceptor responses affect local thermal sensation, static response (affected by the level of skin temperature) and dynamic response (affected by the rate of change of skin temperature). Changes in convective heat transfer due to airflow velocity fluctuations impact the skin temperature fluctuations which result in the local thermal sensation stimuli [5].

\subsection{Airflow velocity fluctuations}

Different studies examined the impact of additional factors affecting the human thermal response to air movement, i.e. whole body thermal sensation [7-10], direction of the airflow [11,12]. Greater attention was however given to the impact of specific dynamic properties of the airflow velocity fluctuations (besides turbulence intensity $\mathrm{Tu}$ ).

Human thermal sensation in the mechanically ventilated indoor environment differs to the naturally ventilated indoor environment. Airflows with properties analogous to the natural airflows were found to be more acceptable [13-15], due to the favourable distribution of the energy of vortices with different frequencies described with power spectrum curve. The slope of the logarithmic power spectrum curve $\beta$ is used in the spectrum analysis. Power spectrum density function $E(f)$ and the power spectrum curve $\beta$ relationship is described with the equation:

$$
E(f)=1 / f^{\beta}
$$

\footnotetext{
* Corresponding author:
} 
where $f$ is the airflow velocity fluctuations frequency (Hz). Typical $\beta$ values range is 0 to -2 . Negative values of $\beta$ from 0 to -0.5 are associated with mechanical airflows, whereas natural winds range typically range from -1.1 to -2.0 , meaning that vortices energy in natural winds is mostly distributed in the low frequency range [16].
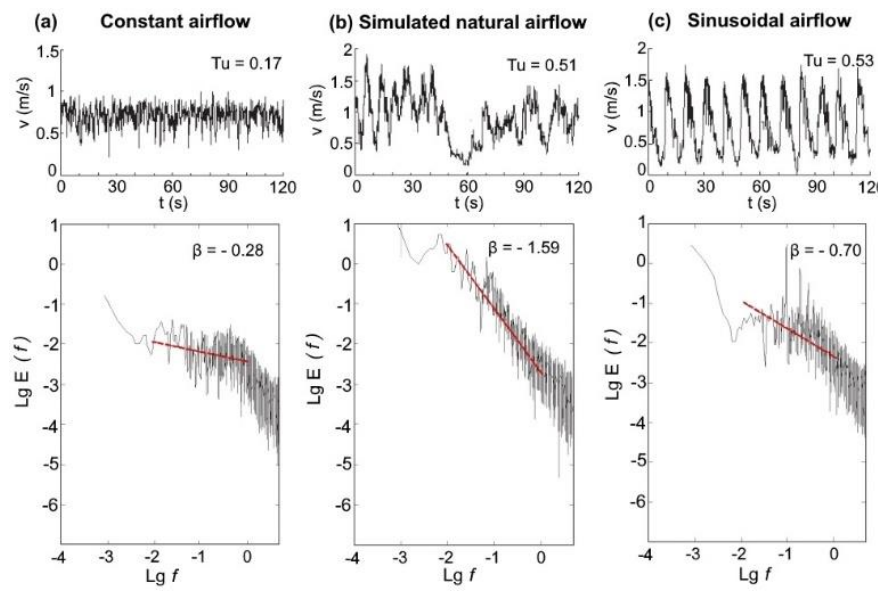

Fig. 1. Air velocity power spectrum curves for different flows; logarithmic power spectrum curve $\beta$ noted in red [15].

Another property of the airflow fluctuations studies is the fluctuating frequency. Several studies found different ranges of frequencies with distinct cooling sensation in the area around $0.5 \mathrm{~Hz}$ [17-22] (Fig. 2).

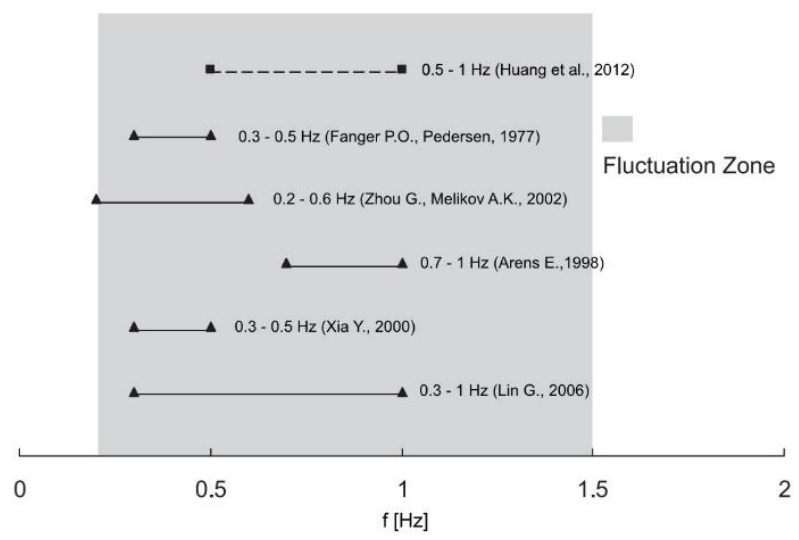

Fig. 2. Fluctuation frequencies with strong cooling effect [17].

\subsection{Airflow temperature fluctuations}

Indoor environment air temperature distribution was studied by Melikov et al. [23] where the impact of temperature fluctuations on human thermal response was accentuated as they anticipated that airflow temperature fluctuations would cause an increased heat flow through the heat receptors below the surface of the skin. Based on the instantaneous measurements of airflow velocities and temperatures they estimated an increase of 0 to $13 \%$ in the heat flow from skin due to temperature fluctuations, which they expected could cause a distinct cooling effect and a feeling of draft.

Madsen and Popiolek [24] also emphasized the likelihood of airflow temperature fluctuations occurring in the indoor environment. They generated an airflow with large temperature fluctuations (standard deviation of $0.8^{\circ} \mathrm{C}$ ) and only $20 \%$ turbulence intensity. By their estimation such an airflow would have a distinct cooling effect, where at least $20 \%$ more people would complain about perceived feeling of draft due to moving air.

In this study aforementioned impact of temperature fluctuations in the airflow on the human thermal response was examined, which has yet to be thoroughly researched in human subjective experiments. Similarly to velocity fluctuations, airflow temperature fluctuations can also arouse dynamic thermoreceptors through rapid changes in the heat flux from the skin and consequently local thermal sensation. For the purposes of the study human subjective experiment was carried out where subjects were exposed to airflows with different fluctuating velocity and temperature characteristics.

\section{Methodology}

\subsection{Experimental plan}

Subjective experiment involved 30 students (Table 1) at the faculty under controlled environmental conditions in the summer of 2016. Subjects were exposed to nonisothermal airflow jets, each with different velocity and temperature fluctuation characteristics. Prior to the experiment the participants were ordered not to consume alcohol or drugs and to attend the experiment well rested so that they were alert during the process.

Table 1. Subjects' profile.

\begin{tabular}{|c|c|c|c|c|}
\hline Gender & $\begin{array}{c}\text { Sample } \\
\text { size }\end{array}$ & Age (years) & Height (cm) & Weight (kg) \\
\hline female & 15 & $26.4 \pm 3.0$ & $165.8 \pm 5.9$ & $57.1 \pm 5.2$ \\
\hline male & 15 & $26.4 \pm 3.3$ & $182.5 \pm 5.1$ & $87.0 \pm 12.9$ \\
\hline
\end{tabular}

Subjects were seated at the desk (sedentary activities, estimated metabolic rate $1.2 \mathrm{met}$ ) and had to be wearing standard summer clothes (clothing insulation value 0.6 clo). Subjects' necks were uncovered during the experiment.

Table 2. Measured ambient conditions.

\begin{tabular}{|l|c|}
\hline air temperature $\left[{ }^{\circ} \mathbf{C}\right]$ & $23.5 \pm 0.2$ \\
\hline relative air humidity [\%] & $50 \pm 5$ \\
\hline mean airflow velocity [m/s] & $0.42 \pm 0.02$ \\
\hline measured PMV [/] & $0.60 \pm 0.10$ \\
\hline
\end{tabular}

Measured ambient conditions during the experiment are shown in Table 2. Instantaneous records of airflow velocity and temperature were measured with thermoanemometer (Swema 03+ thermoanemometer, Swema AB, Sweden), positioned $20 \mathrm{~cm}$ from the exit of the air supply duct. The sampling frequency was $10 \mathrm{~Hz}$ 
(velocity precision $\pm 5 \%$, temperature precision $\pm 0.3^{\circ} \mathrm{C}$ ). Subjects' necks were positioned $30 \mathrm{~cm}$ from the exit of the air duct (Fig. 3). PMV and PPD indices meter (AM101, Kyoto Electronics Manufacturing Co. Ltd., Japan) was positioned at the subjects' desk to measure the ambient conditions and PMV index during the experiment.

Subjects were exposed to 6 airflows which differentiated in amplitude and frequency of temperature fluctuations. Exposure time for each airflow was 180 seconds. After this period, subjects received a questionnaire in relation to their thermal sensation and perceptive airflow intensity of the air movement.

\subsection{Airflow handling unit}

Airflow handling unit (Fig. 3) was designed as to allow generating jets with fluctuating temperature. At the inlet duct supply air entered the unit (light blue arrow) through which it reached the airflow separator box, which divided the jet in two equal parts. After the separator box one part of the airflow continued along the duct with air cooler and the other half continued along the duct with air heater. Airflows were again connected in a Y-piece duct section. Level part of Y-piece was partitioned down the middle so the heated and cooled airflows stayed separated. Stepper motor was positioned at the end of the partition. Motor shaft had a $2 / 3$ circular turntable attached which alternately let through heated and cooled airflow at the end of the airflow supply duct. The stepper motor (and circular turntable) rotational speed was controlled through a computer which resulted in different frequencies of the alternating cold and hot airflow. By alternating the two airflows temperature fluctuation frequencies and amplitudes were adjusted.

\subsection{Generated airflow}

Subjects were exposed to 6 different airflows. Generated airflow velocity and temperature properties are presented in Table 2. Since both velocity and temperature fluctuations were dependant on the turntable rotational speed they both coincided and had similar sinusoidal characteristics (Fig. 4). Main difference between the airflows was in the amplitude and frequency of the velocity and temperature fluctuations.

Table 3. Airflow velocity and temperature characteristics.

\begin{tabular}{|l|c|c|c|c|c|c|}
\hline Airflow & $\mathbf{1}$ & $\mathbf{2}$ & $\mathbf{3}$ & $\mathbf{4}$ & $\mathbf{5}$ & $\mathbf{6}$ \\
\hline $\begin{array}{l}\text { Turntable rotating } \\
\text { frequency [Hz] }\end{array}$ & 0.2 & 0.25 & 0.3 & 0.5 & 1 & 4 \\
\hline $\begin{array}{l}\text { Fluctuation frequency } \\
\text { (velocity, temp.) [Hz] }\end{array}$ & 0.028 & 0.033 & 0.039 & 0.062 & 0.126 & 0.5 \\
\hline $\begin{array}{l}\text { Velocity fluctuations } \\
\text { SD [m/s] }\end{array}$ & 0.21 & 0.21 & 0.21 & 0.21 & 0.22 & 0.16 \\
\hline $\begin{array}{l}\text { Turbulence intensity } \\
\text { [\%] }\end{array}$ & 48.87 & 50.64 & 48.90 & 50.57 & 50.61 & 38.44 \\
\hline $\begin{array}{l}\text { Logarithmic power } \\
\text { spectrum curve } \boldsymbol{\beta}[/]\end{array}$ & -0.64 & -0.66 & -0.62 & -0.63 & -0.66 & -0.49 \\
\hline $\begin{array}{l}\text { Average fluctuating } \\
\text { temp. amplitude [ }{ }^{\circ} \mathbf{C} \text { ] }\end{array}$ & 1.65 & 1.40 & 1.30 & 0.90 & 0.58 & 0.31 \\
\hline $\begin{array}{l}\text { SD of temperature } \\
\text { fluctuations [ }{ }^{\circ} \mathbf{C} \text { ] }\end{array}$ & 0.50 & 0.43 & 0.36 & 0.26 & 0.13 & 0.06 \\
\hline
\end{tabular}

By increasing turntable rotation frequency, temperature fluctuations amplitude and SD decreased (Table 3, Fig. 4).

Highest fluctuating frequencies were noted at the highest turntable frequency whereas lowest rotating frequencies resulted in highest amplitudes.

Turbulent behaviour as a result of mixing jets at the end of the airflow supply duct is reflected in high turbulence intensity values, i.e. between $40 \%$ to $50 \%$.

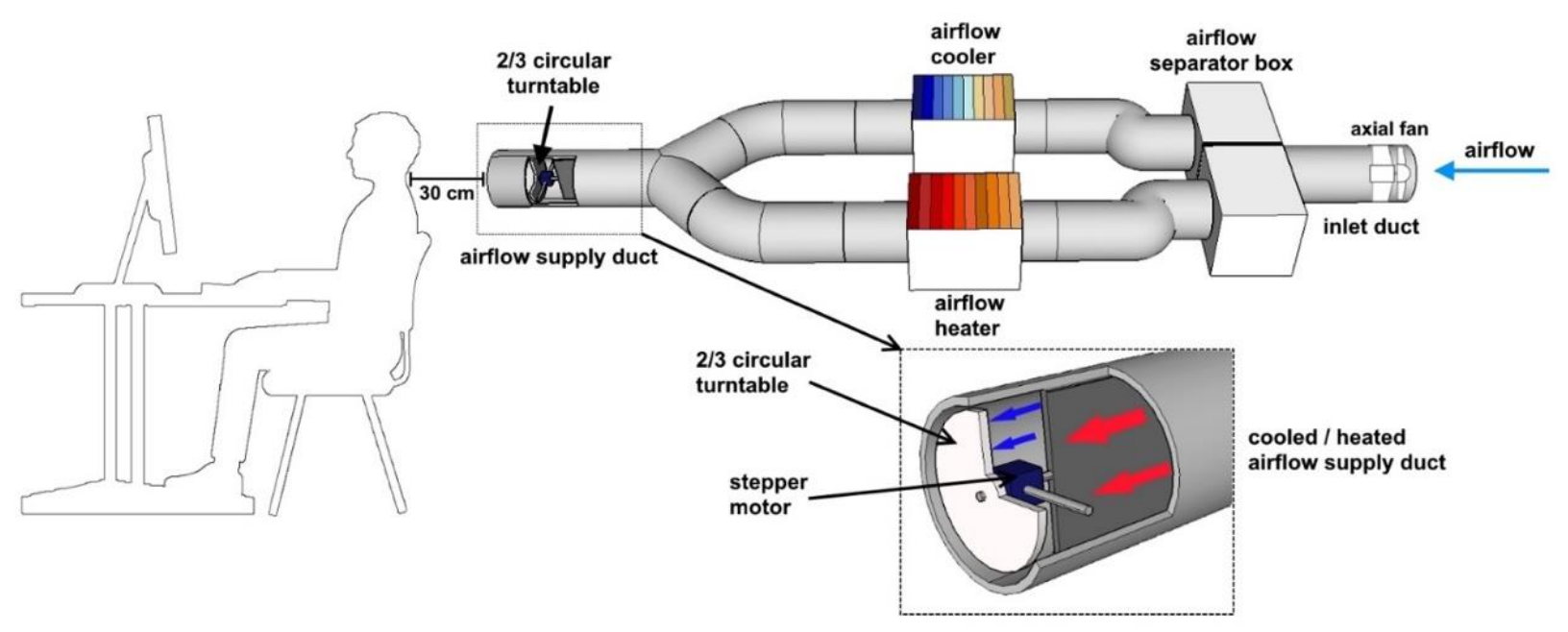

Fig. 3. Experiment scheme and airflow handling unit 
While with increasing turntable rotating frequency the amplitude and standard deviation of temperature fluctuations diminished, turbulence intensity remained rather high throughout the rotating frequency spectrum.
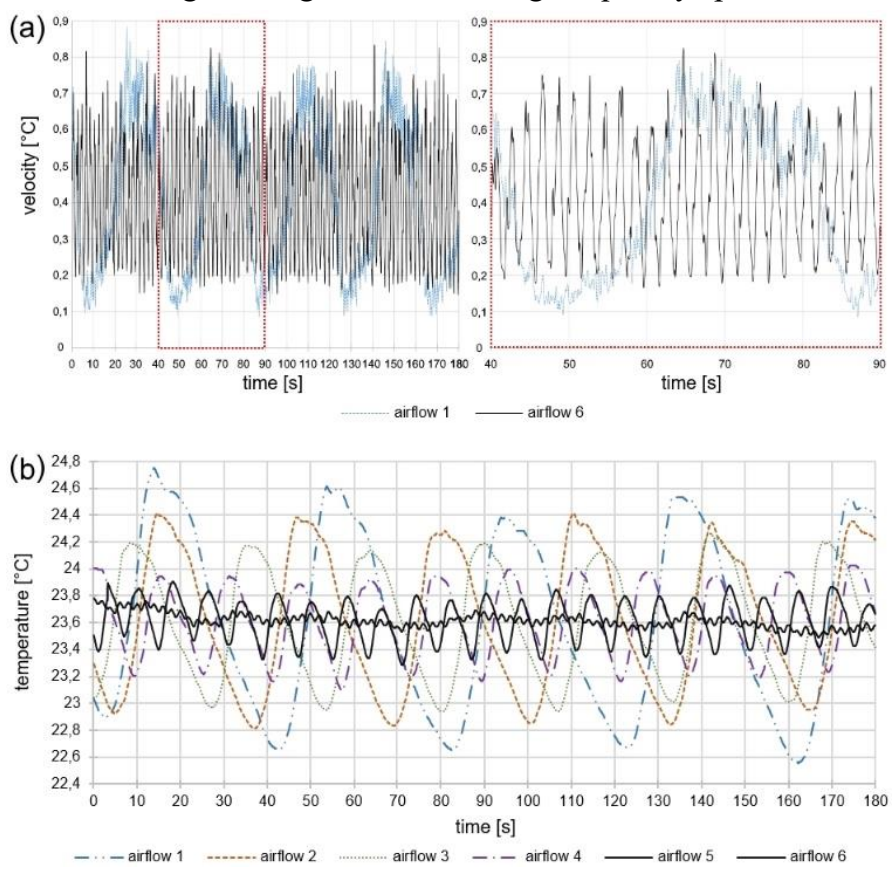

Fig. 4. Airflows' velocity (a) and temperature (b) time series.

Power spectrum density curves for the airflows were analysed as well. For airflows 1 to 5 registered $\beta$ values are around -0.65 ; given the sinusoidal velocity series registered $\beta$ values coincide with results from other studies [15]. For the airflow 6 the $\beta$ value is the lowest -0.49 with characteristics resembling that of a constant airflow with fairly evenly distributed energy of vortices across the frequency spectrum.
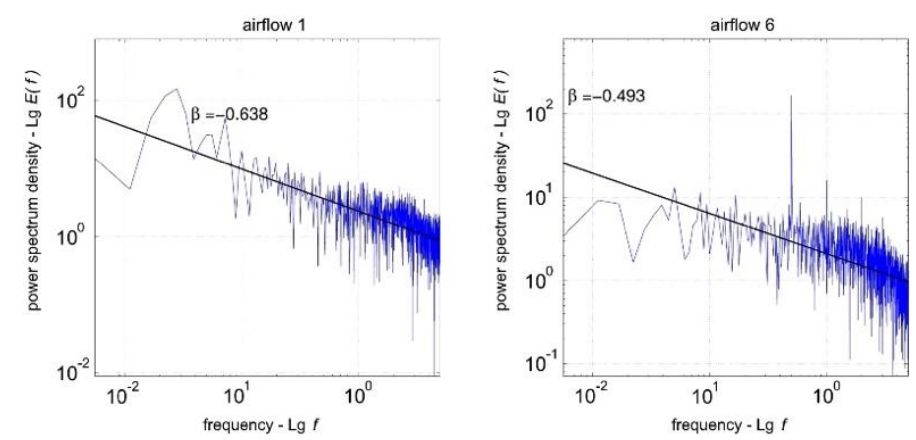

Fig. 5. Power spectrum density curves (airflows 1, 6).

\subsection{Heat flux changes for generated airflows}

Melikov [25] as well as Madsen and Popiolek [24] analysed changes in the heat flux, calculated with and without taking into account airflow temperature fluctuations. Based on the instantaneous records of velocity and temperature similar analysis was carried for generated airflows in this study as well. The instantaneous heat flux was calculated by:

$$
q=\left(t_{s}-t_{a}\right) \cdot h_{c}\left[W / m^{2}\right]
$$

where $t_{s}$ is the skin temperature, $t_{a}$ is the air temperature and $h_{c}$ is a convective heat transfer coefficient. Convective heat transfer coefficient was calculated by:

$$
h_{c}=a \cdot v^{b}\left[W / m^{2} K\right]
$$

where $v$ is the air velocity and $a$ and $b$ are constants for which values ( $a=8.3, b=0.6)$ were taken from [26].

Firstly the instantaneous heat flux was calculated with instantaneous velocity records and mean air temperature (for the measured airflow) and secondly using both instantaneous velocity and temperature records. Changes for calculated values of mean heat fluxes $\bar{q}$ and its standard deviations $\mathrm{SD}_{\bar{q}}$ for generated airflows are shown in Table 4.

Table 4. Mean heat flux and standard deviation changes.

\begin{tabular}{|l|c|c|c|c|c|c|}
\hline airflow & $\mathbf{1}$ & $\mathbf{2}$ & $\mathbf{3}$ & $\mathbf{4}$ & $\mathbf{5}$ & $\mathbf{6}$ \\
\hline$\Delta \bar{q}[\%]$ & 0.48 & 0.54 & 0.36 & 0.04 & -0.03 & -0.01 \\
\hline$\Delta \mathrm{SD} \bar{q}[\%]$ & 6.27 & 4.88 & 3.90 & 1.57 & -0.08 & -0.23 \\
\hline
\end{tabular}

For all the generated airflows changes in mean heat flux are minimal (less than 1\%) when comparing calculations with and without considering temperature fluctuations. Similar small changes can be observed for the the standard deviation. Melikov [25] as well as Madsen and Popiolek [24] observed changes in heat flux by up to $13 \%$ which they suggested affected the increase in heat flow from the skin and therefore increased cooling effect. In our case the increased heat flux was not achieved as expected since the generated airflows had sinusoidal characteristics around the mean temperature.

\section{Results of subjective experiment}

The subjective experiment results are grouped based on the results of the exposure to one of the six airflows with different velocity and temperature properties.

\subsection{Thermal comfort vote}

At the first, the subjects were asked about their thermal comfort during the exposure to airflows ( -3 very cold, +3 hot, 0 neutral). During the exposure the predicted mean vote was also measured with a PMV index meter.

Thermal comfort vote (TCV) values for all the airflows are shown in figure 6 . Comfort votes ranged between -0.83 to -0.43 , i.e. in the range between "slightly cool" and "cool", which is in contrast to the measured value of PMV $(0.60 \pm 0.10)$ which suggested a "slightly warm" thermal environment.

Subjects expressed distinct cooling sensation when exposed to the airflows, most noticeable for airflows 1 and 6 (airflows with highest temperature fluctuating amplitudes or frequencies). For airflows 2 to 5 subjects returned slightly higher votes, around the value -0.5 . 


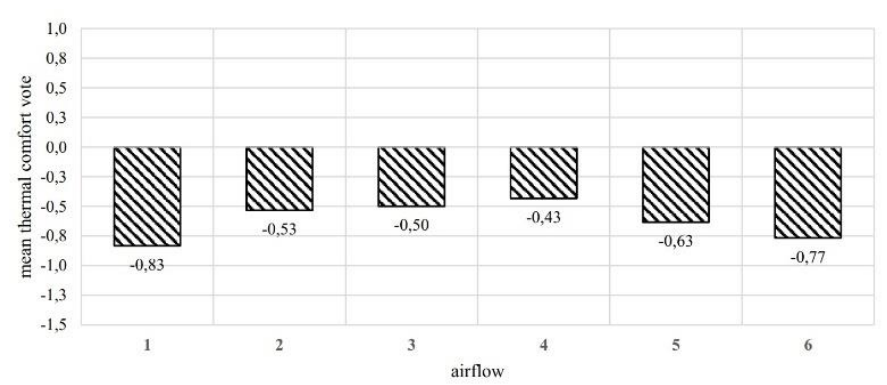

Fig. 6. Mean thermal comfort vote when exposed to airflows.

\subsection{Airflow intensity}

At the second survey question, subjects had to answer, on the scale of 1 to 5 , about the intensity of the airflow, i.e. how strongly the participants felt the air movement (Fig. 7). Again, the highest values were recorded for airflows 1 and 6 (3.10 and 3.03 respectively), while the recorded values for airflows 2 to 5 were a tad lover (2.40 to 2.77).

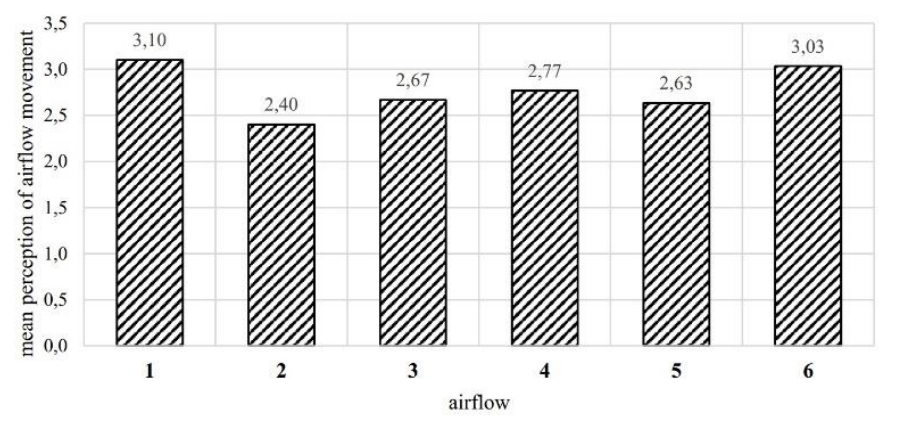

Fig. 7. Mean votes for airflow intensity.

\section{Discussion and conclusions}

Dynamic response of skin thermoreceptors is related to changes in skin temperature [6], which are affected by convective heat transfer. Similarly to airflow velocity fluctuations, temperature fluctuations were investigated in this study with a goal to evaluate consequent affects to local thermal sensation.

An air handling unit was constructed for producing airflows with fluctuating temperature and used in a laboratory experiment, involving 15 female and 15 male young adults. The participants had to complete a questionnaire regarding their thermal comfort sensation and the felt airflow intensity, after being exposed to 6 airflows with different fluctuating velocity and temperature characteristics (Table 3 ).

The thermal comfort votes and airflow intensity perception analysis suggested a distinct cooling effect on the subjects due to airflows (Figure 6). The mean TCV value ranged from -0.43 to -0.83 . Based on the TCV results, participants experienced a "slightly cool" thermal sensation during the exposure to air movement, which outweighed the "slightly warm" thermal environment(measured PMV value $0.60 \pm 0.10$ during the experiment). Participants felt the strongest cooling effect for airflows with the highest values of temperature amplitude and frequency fluctuations (airflows 1 and 6).

When taking into account the comfort votes and the air movement intensity perception it could be presumed that airflows 2 to 5 were more favourable to the subjects, especially with regard to the "slightly warm" thermal environment. Airflows 1 and 6, however, were slightly less favourable for the subjects, as indicated by the cooler thermal comfort votes and the more intense perception of air movement.

As pointed out in earlier studies on dynamic airflow properties, a strong cooling effect can be a result of specific airflow fluctuating frequencies (Section 1.3). As can be seen in Figure 2, airflow velocity fluctuations in the range of 0.2 and $1 \mathrm{~Hz}$ have a stronger cooling effect than airflows at other frequencies. Airflow $6(0.5 \mathrm{~Hz}$ fluctuating frequency) fits into this range. For this reason, we cannot contribute the elevated airflow sensitivity only to temperature fluctuations. An elevated level of sensitivity (as well as the distinct cooling sensation) to the airflow was also noted at airflow 1, where the frequency of velocity fluctuations was outside the $0.2-1 \mathrm{~Hz}$ range. In this case, the pronounced cooling effect and the increased sensitivity to the air movement could be linked with temperature fluctuations.

The potential increased cooling effect of airflows with temperature fluctuations could be used in the future for air-conditioning systems in indoor environment. In this study, an increased cooling effect was achieved even though there were no changes to the heat flux (Section 2.4). For the practical use in the indoor environment, future studies should focus on possible satisfactory combinations of temperature (and velocity) fluctuations in the airflow for comfortable thermal environment at elevated temperatures. Mixing smaller air rates of cooled air with warmer indoor air could result in energy savings while still achieving satisfactory thermal conditions.

One also has to consider the potential negative effects of airflow temperature fluctuations on thermal sensation. An increased cooling effect can result in the occurrence of draft. While the current study concentrated only on possible beneficiary effects of airflow temperature fluctuations on individuals, attention should also be paid to the potential risk of draft perception.

Due to the small sample size, statistical significance of experimental results was not achieved. Nevertheless, the experimental results and theoretical assumptions regarding the dynamic response of thermoreceptors demonstrate implications for further research into the impact of temperature fluctuations on the perception of air movement. 


\section{References}

[1] N.E. Klepeis, W.C. Nelson, W.R. Ott, J.P. Robinson, A.M. Tsang, P. Switzer, J. V. Behar, S.C. Hern, W.H. Engelmann, The National Human Activity Pattern Survey (NHAPS): A resource for assessing exposure to environmental pollutants, J. Expo. Anal. Environ. Epidemiol. 11 (2001) 231-252. doi:10.1038/sj.jea.7500165.

[2] F.C. Houghten, C. Gutberlet, E. Witkowski, Draft Temperatures and Velocities in Relation to Skin Temperature and Feeling of Warmth., Heating, Pip. Air Cond. 10 (1938) 145-152.

[3] L.G. Berglund, A.P.R. Fobelets, Subjective human response to low-level air currents and asymmetric radiation, in: ASHRAE Trans., 1987: pp. 497-523.

[4] P.O. Fanger, N.K. Christensen, Perception of draught in ventilated spaces, Ergonomics. 29 (1986) 215-235. doi:10.1080/00140138608968261.

[5] P.O. Fanger, A.K. Melikov, H. Hanzawa, J. Ring, Air turbulence and sensation of draught, Energy Build. 12 (1988) 21-39. doi:10.1016/0378-7788(88)90053-9.

[6] H. Hensel, K. Schafer, Thermoreception and Temperature Regulation in Man, in: E.F.J. Ring, B. Phillips (Eds.), Recent Adv. Med. Thermol., Springer, Boston, MA, 1984: pp. 51-64.

[7] J. Toftum, A. Melikov, A. Tynel, M. Bruzda, P.O. Fanger, Human response to air movement Evaluation of ASHRAE's Draft Criteria (RP843), HVAC \&R Res. 9 (2003) 187-202. doi:10.1080/10789669.2003.10391064.

[8] J. Toftum, Air movement--good or bad?, Indoor Air. 14 Suppl 7 (2004) 40-45. doi:10.1111/j.1600-0668.2004.00271.x.

[9] J. Palonen, O. Seppänen, Design criteria for central ventilation and air-conditioning system of offices in cold climate, in: Indoor Air '90, Proc. 5th Int. Conf. Indoor Air Qual. Clim., Toronto, Canada, 1990: pp. 299-305.

[10] J. Toftum, R. Nielsen, Draught sensitivity is influenced by general thermal sensation, Int. J. Ind. Ergon. 18 (1996) 295-305. doi:10.1016/0169-8141(95)00070-4.

[11] G. Zhou, Human Perception of Air Movement: Impact of Frequency and Airflow Direction on Draught Sensation, International Centre for Indoor Environment and Energy, Department for Energy Engineering, Technical University of Denmark, 1999. https://books.google.si/books?id=UUYlQwAAC AAJ.

[12] J. Toftum, G. Zhou, A.K. Melikov, Effect of airflow direction on human perception of draught, Clima 2000. 366 (1997) 1-12. https://pdfs.semanticscholar.org/3a9b/c78141659 022d3e207879b65a240c9820ea4.pdf.

[13] Q. Jia, Study on dynamization of air supply terminal, Tsinghua University, Beijing, 2000.

[14] W. Cui, G. Cao, Q. Ouyang, Y. Zhu, Influence of dynamic environment with different airflows on human performance, Build. Environ. 62 (2013) 124-132. doi:10.1016/j.buildenv.2013.01.008.

[15] X. Zhou, Q. Ouyang, G. Lin, Y. Zhu, Impact of dynamic airflow on human thermal response, Indoor Air. 16 (2006) 348-355. doi:10.1111/j.1600-0668.2006.00430.x.

[16] Y. Zhu, M. Luo, Q. Ouyang, L. Huang, B. Cao, Dynamic characteristics and comfort assessment of airflows in indoor environments: A review, Build. Environ. 91 (2015) 5-14. doi:10.1016/j.buildenv.2015.03.032.

[17] L. Huang, Q. Ouyang, Y. Zhu, Perceptible airflow fluctuation frequency and human thermal response, Build. Environ. 54 (2012) 14-19. doi:10.1016/j.buildenv.2012.02.004.

[18] G. Zhou, A.K. Melikov, P.O. Fanger, Impact of equivalent frequency on the sensation of draught, in: Proc. Roomvent 2002, 2002: pp. 297-300.

[19] S. Tanabe, K. Kimura, Importance of air movement for thermal comfort under hot and humid conditions, Proc. Second ASHRAE Far East Conf. Air Cond. Hot Clim. Kuala Lumpur, Malaysia. (1989) 95-103.

[20] E. Arens, T. Xu, K. Miura, Z. Hui, M. Fountain, F. Bauman, A study of occupant cooling by personally controlled air movement, Energy Build. 27 (1998) 45-59. doi:10.1016/S03787788(97)00025-X.

[21] Y. Xia, Effect of air turbulent intensity and frequency on human thermal sensation, Tsinghua University, 2000.

[22] G. Lin, Effect of airflow frequency on human thermal response, Tsinghua University, 2006.

[23] A.K. Melikov, U. Krüger, G. Zhou, T.L. Madsen, G. Langkilde, Air Temperature Fluctuations in Rooms, Build. Environ. 32 (1997) 101-114.

[24] T.L. Madsen, Z. Popiolek, An instrument for draught measurements caused by fluctuations in air temperature and/or velocity., in: Proc. Roomvent'94, Air Distrib. Rooms Fourth Int. Conf., 1994.

[25] A. Melikov, U. Krüger, G. Zhou, T.. Madsen, G. Langkilde, Air temperature fluctuations in rooms, Build. Environ. (1997).

[26] American society of Heating Refrigerating, Engineers Air-Conditioning, ASHRAE handbook. Fundamentals, [SI ed.], Atlanta, Ga. : American Society of Heating, Refrigerating, and Air-Conditioning Engineers, 1996. 